LONGINES appartient depuis bientôt un siècle à l'élite des fabriques suisses d'horlogerie et prouve chaque jour ses traditions de qualité et de précision.

\title{
La montre
}

LONGINES jouit d'un rayonnement inégalé dans 150 pays, justifiant pleinement l'appellation des Anglo-Saxons...

THE WORLD'S MOST HONOURED WATCH

\section{IL EST TEMPS... LONGINES}

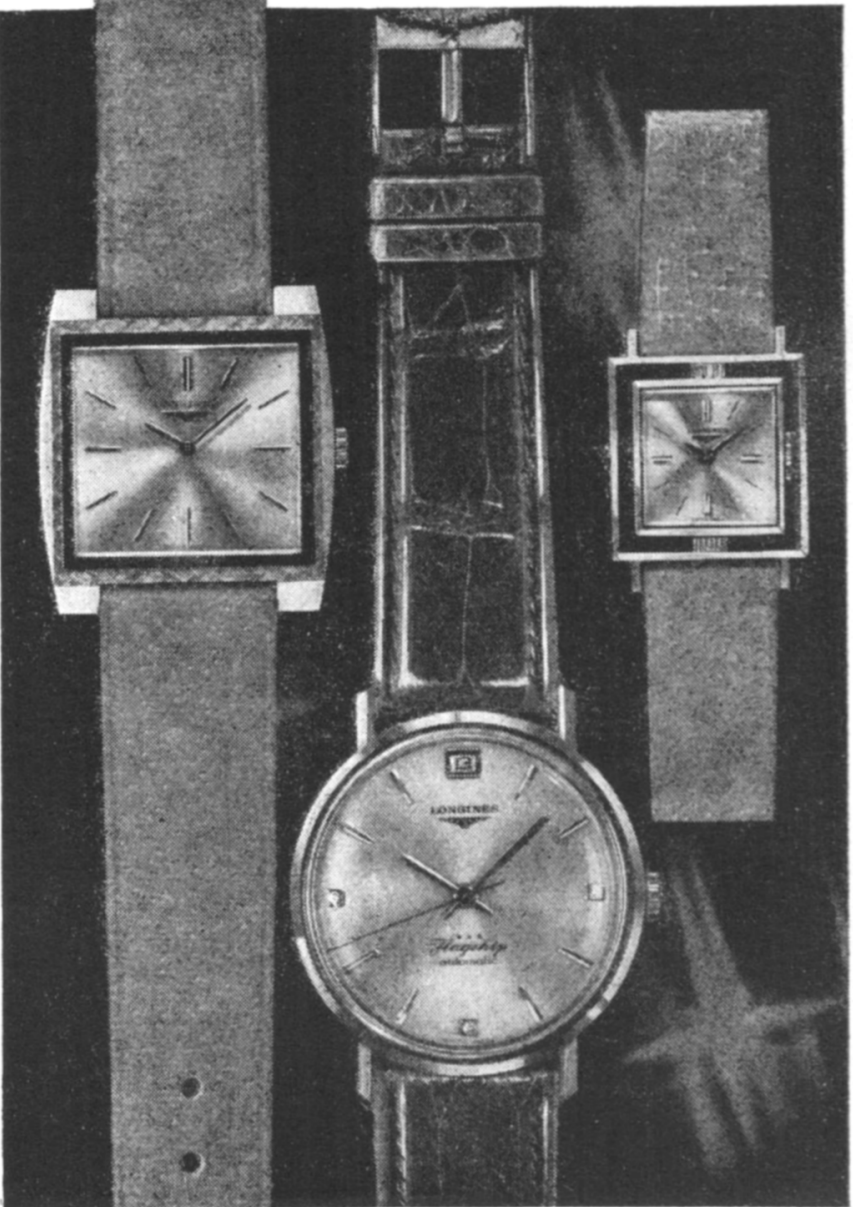

Rèf. 7337 Boitier forme tonneau; or $18 \mathrm{ct}$. Lunette lapidée, décor tuscanisé, Fr. 775.- .

Réf. 3508 Flagship $* \star \star$ Automatic, calendrier, De Luxe; or $18 \mathrm{ct}$. cadran or massif avec brillants. Fr. 1090.- .

Réf. 7368 Boitler carré, avec cadre ajouré; or 18 ct. Fr. 765.-. 


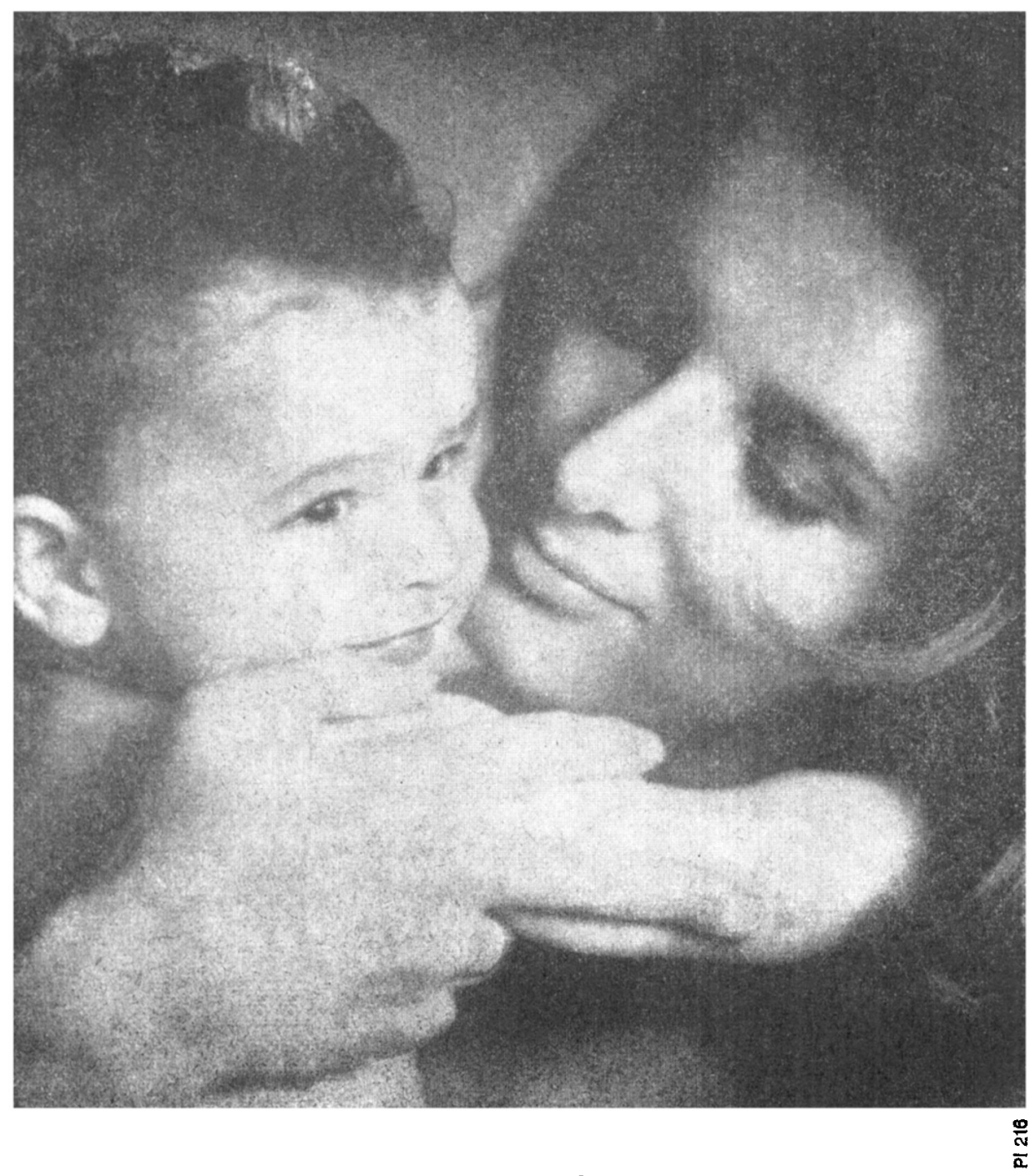

\section{Depuis 100 ans, Nestlé au service de l'enfance dans toutes les parties du monde.}

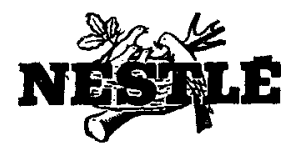



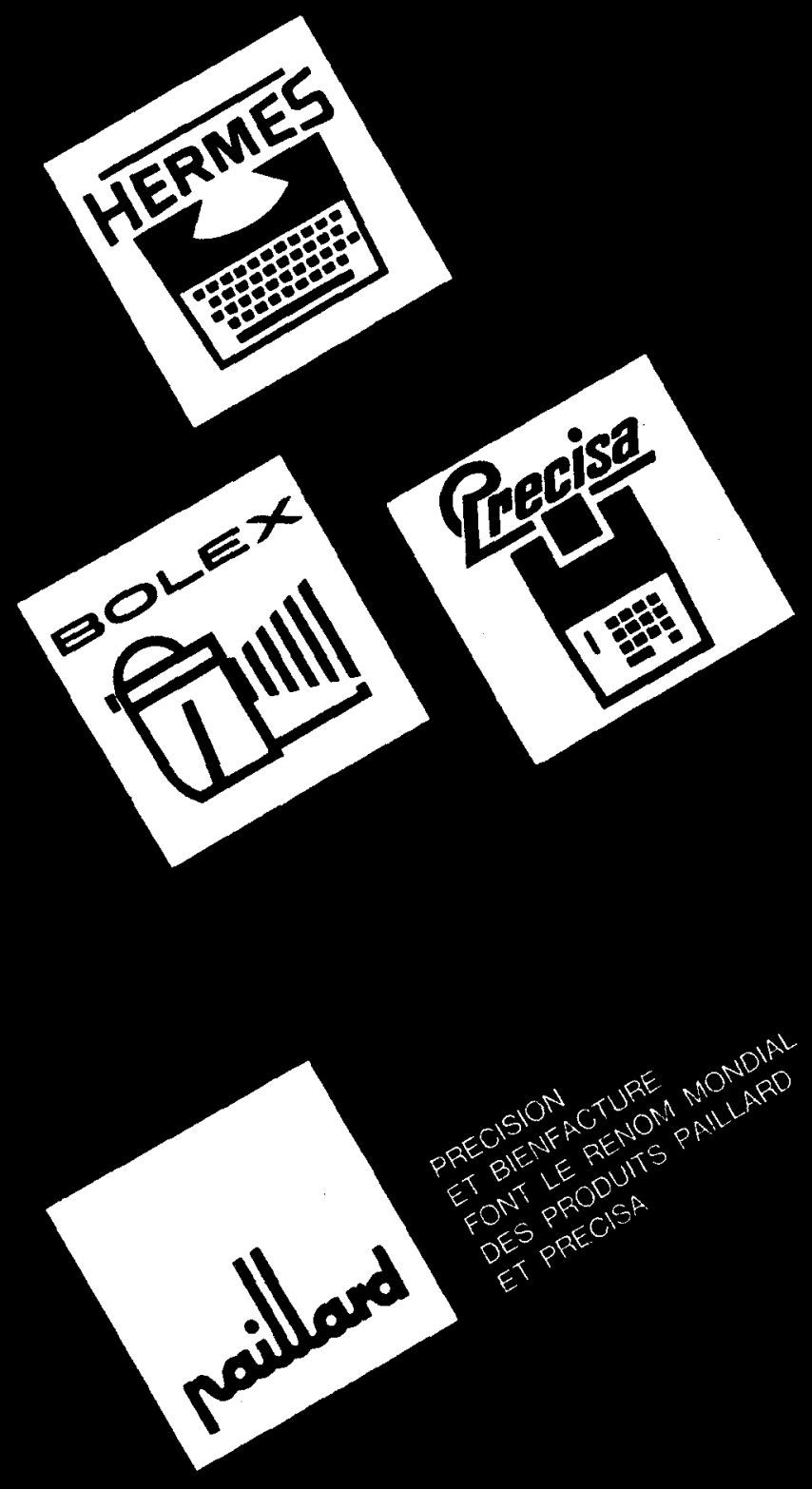


\section{Produits}

\section{pharmaceutiques}

\section{SANDOZ}

\section{en toute}

\section{sécurité}

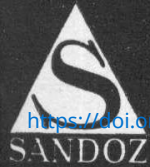



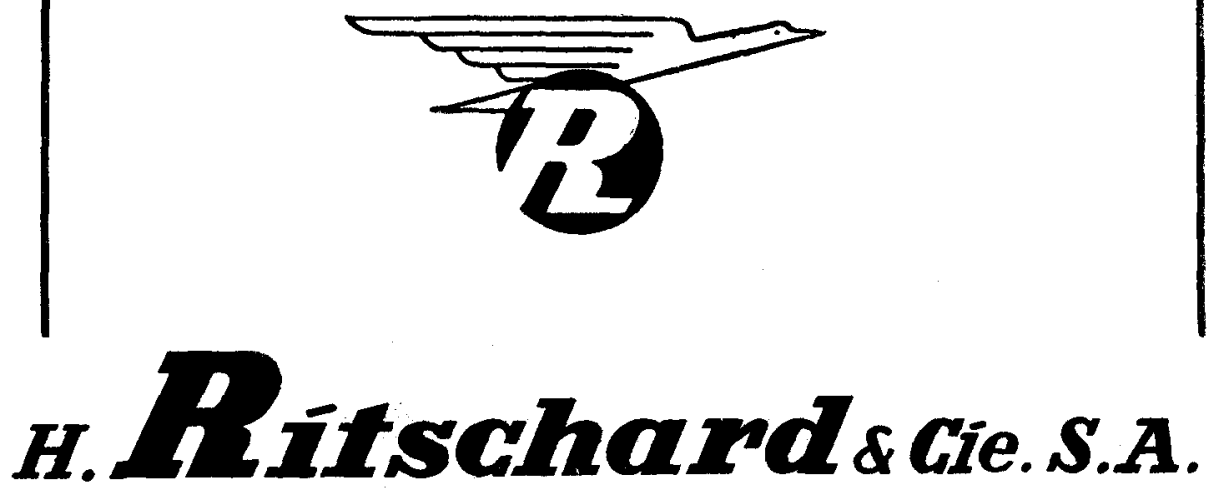

\title{
TRANSPORTS INTERNATIONAUX
}

\author{
AGENCE DE VOYAGES \\ GENEV VE, 18, place Cornavin \\ Téléphones 323400 et 323402 - Télex 22167
}

Change - Billets - Passages maritimes

Assurances - Agence en douane

Camionnages - Entrepôts

Livraison à domicile des billets avion et chemin de fer sur simple commande téléphonique

Succursales :

LAUSANNE - BALE - ZURICH - ANNEMASSE (France) 

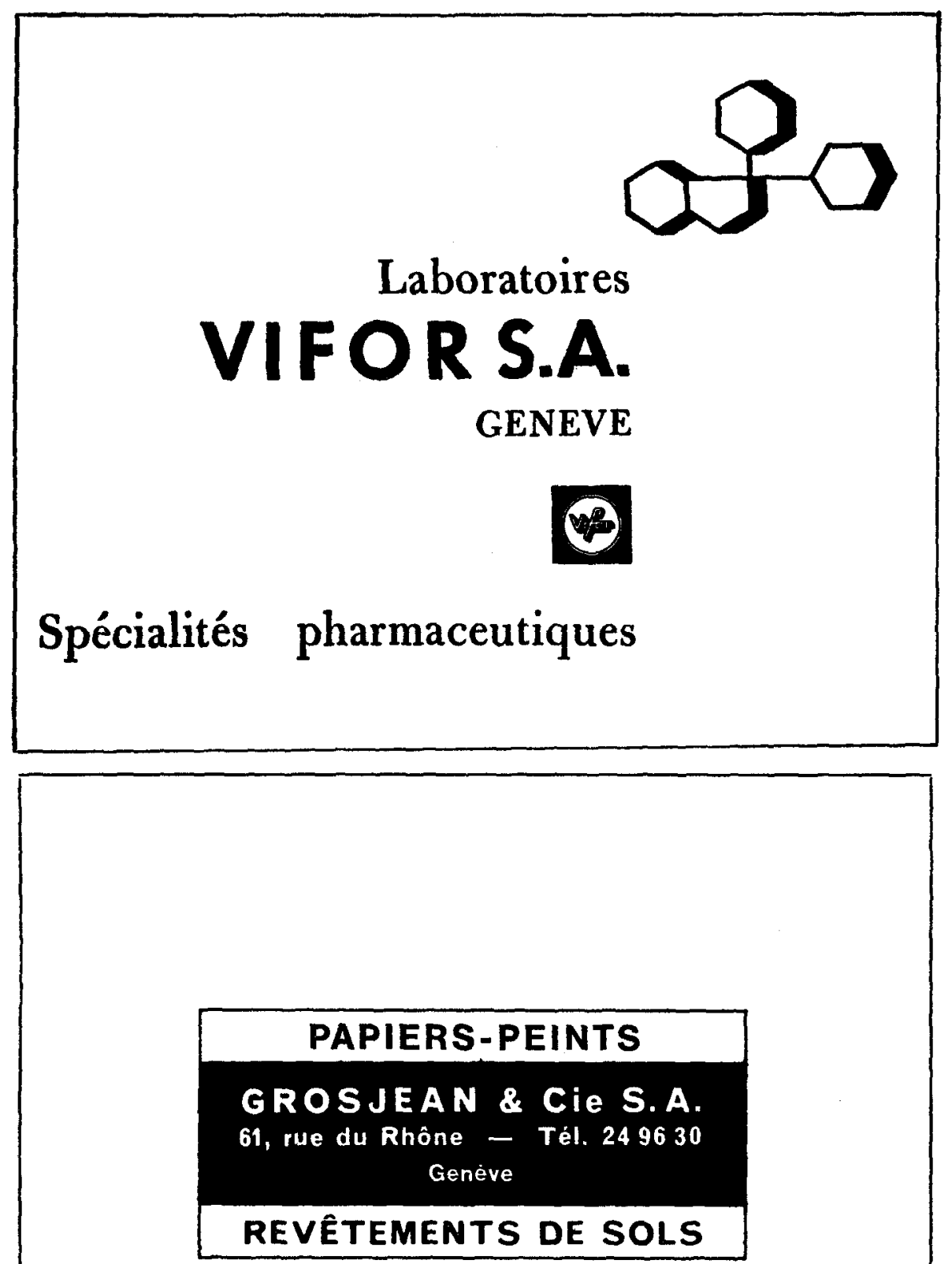


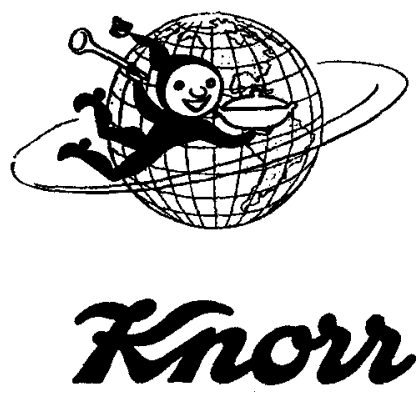

synonyme de tradition

et de qualité

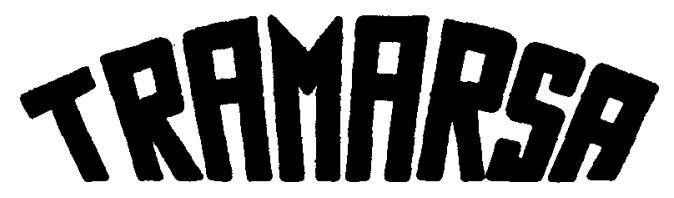

- transports internationaux

voyages

GENÈVE (sièce)

17, rue du Mt-Blanc

Tól. 312135
BALE

149 Spalenring

Tél. 235074
LE LOCLE SION

Tét. 51684

T6l. 24823

ZURICH 109 Militărstr. Tól. 237888 


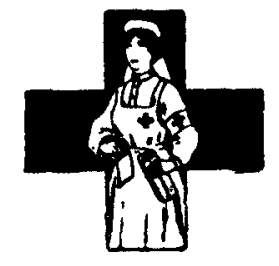

A L'ambulahge

M. BOVARD - GENEVE

16, PLAOE DES PHHOEOPHES

\section{FabRique d'OBJETS DE Pansement}

MOBILIER MÉDICAL - INSTRUMENTS DE CHIRURGIE APPAREILS ÉLECTRO-MÉDICAUX
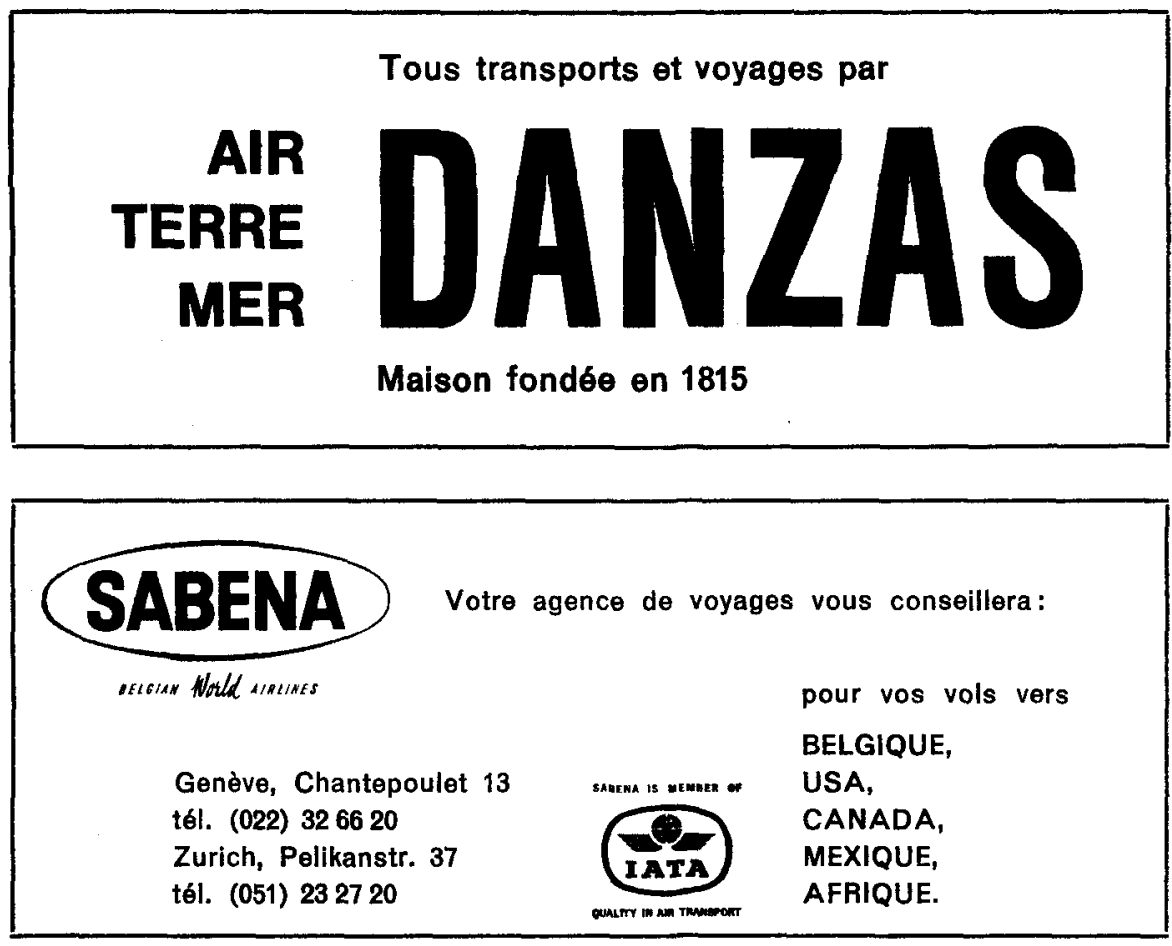

Montex S.a.

Exportation - Importation
4, rue du Mont-Blanc

\section{Genève}

Téléphone: (022) 326612

Télex 23458

Cables : Corvin Genève 


\section{EXTRAIT DES STATUTS \\ DU COMITE INTERNATIONAL DE LA CROIX-ROUGE (adoptés le 25 septembre I952)}

Article premrer. - Le Comité international de la Croix-Rouge (CICR), fondé à Genève en 1863 , consacré par les Conventions de Geneve et par les Conférences internationales de la Croix-Rouge, est une institution indépendante ayant son statut propre.

Il est partie constitutive de la Croix-Rouge internationale 1.

ART. 2. - En tant qu'association régie par les articles 60 et suivants du Code civil suisse, le CICR possède la personnalité civile.

Art. 3. - Le CICR a son siège à Genève.

Il a pour emblème la croix rouge sur fond blanc. Sa devise est a Inter arma caritas $n$.

ArT. 4. - Le CICR a, notamment, pour rôle :

a) de maintenir les principes fondamentaux et permanents de la CroixRouge, à savoir: l'impartialité, une action indépendante de toute considération raciale, politique, confessionnelle ou économique, l'universalité de la Croix-Rouge et l'égalité des Sociétés nationales de la Croix-Rouge ;

b) de reconnaître toute Société nationale de la Croix-Rouge nouvellement créé ou reconstituée et répondant aux conditions de reconnaissance en vigueur, et de notifier cette reconnaissance aux autres Sociétés nationales;

c) d'assumer les tâches qui lui sont reconnues par les Conventions de Genève, de travailler à l'application fidèle de ces Conventions et de recevoir toute plainte au sujet de violations alléguées des Conventions humanitaires ;

d) d'agir, en sa qualité d'institution neutre, spécialement en cas de guerre, de guerre civile ou de troubles intérieurs; de s'employer en tout temps à ce que les victimes militaires et civiles desdits conflits et de leurs suites directes reçoivent protection et assistance, et de servir, sur le plan humanitaire, d'intermédiaire entre les parties;

e) de contribuer, en vue desdits conflits, à la préparation et au développement du personnel et du matériel sanitaires, en collaboration avec les organisations de la Croix-Rouge et les Services de santé militaires et autres autorités compétentes;

f) de travailler au perfectionnement du droit international humanitaire, à la compréhension et la diffusion des Conventions de Genève et d'en préparer les développements éventuels ;

g) d'assumer les mandats qui lui sont confiés par les Conférences internationales de la Croix-Rouge.

Le CICR peut en outre prendre tonte initiative humanitaire qui entre dans son róle d'institution spécifiquement neutre et indépendante et étudier toute question dont l'examen par une telle institution s'impose.

ART. 6 (alinéa premier). - Le CICR se recrute par cooptation parmi les citoyens suisses. Le nombre de ses membres ne peut dépasser vingt-cinq.

1 La Croix-Rouge internationale comprend les Sociétés nationales de la Croix-Rouge, le Comité international de la Croix-Rouge et la Ligue des Sociétés de la Croix-Rouge. L'expression a Sociétés nationales de la Croix-Rouge » couvre également les Sociétés du Croissant-Rouge et la Société du Lion-et-Soleil-Rouge. 


\section{ADRESSES DES COMITÉS CENTRAUX}

AFGHANISTAN - Croissant-Rouge afghan, Kaboul.

AFRIQUE DU SUD (République) - Croix-Rouge sud-africaine, Cor. Kruis \& Market Streets, P.O.B. 8726, Johannesburg.

ALBANIE - Croix-Rouge albanaise, 35, Rruga e Barrikadavet, Tirana.

ALGÉRIE - Comité central du Croissant-Rouge algérien, 15 bis, boulevard Mohamed V, Alger.

ALLEMAGNE (République démocratique) - CroixRouge allemande dans la République démocratique allemande, 2, Kaitzerstrasse, Dresde $A 1$.

ALLEMAGNE (République fédérale) - CroixRouge allemande dans la République fédérale d'Allemagne, 71, Friedrich-Ebert-Allee, 5300 Bonn 1, Postfach.

ARABIE SÉOUDITE - Croissant-Rouge de l'Arabie Séoudite, Riyad.

RÉPUBLIQUE ARABE UNIE - Croissant-Rouge de la République Arabe Unie, rue Ramsès, 34, Le Caire.

ARGENTINE - Croix-Rouge argentine, H. Yrigoyen 2068, Buenos - Aires.

AUSTRALIE - Croix-Rouge australienne, 122-128 Flinders Street, Melbourne, C.I.

AUTRICHE - Croix-Rouge autrichienne, 3, Gusshausstrasse, Postfach 39, Vienne IV.

BELGIQUE - Croix-Rouge de Belgique, 98, chaussée de Vleurgat, Bruxelles 5.

BIRMANIE - Croix-Rouge de Birmanie, 42, Strand Road, Red Cross Building, Rangoon.

BOLIVIE - Croix-Rouge bolivienne, avenue SimonBolivar, 1515 (Casilla 741), La Paz.

BRÉSIL - Croix-Rouge brésilienne, Praça da Cruz Vermelha, 10-12, Rio de Janeiro.

BULGARIE - Croix-Rouge bulgare, 1, boul. S. S. Biruzov, Sofia.

BURUNDI - Croix-Rouge du Burundi, B.P. 324 rue du Marché 3, Bujumbura.

CAMBODGE - Croix-Rouge cambodgienne, $17 \mathrm{R}$ Vithei Croix-Rouge cambodgienne, B.P. 94, Plinom-Penh.

CAMEROUN - Comité central de la Croix-Rouge camerounaise, rue Henri-Dunant, Boîte postale 631, Yaoundé.

CANADA - Croix-Rouge canadienne, 95, Wellesley Street East, Toronto, 5.

CEYLAN - Croix-Rouge de Ceylan, 106, Dharmapala Mawatte, Colombo VII.

CHILI - Croix-Rouge chilienne, Avenida Santa Maria, 0150 Correo, 15, Casilla 246.V., Santiago de Chile.

CHINE - Croix-Rouge chinoise, 22, Kanmien Hutung, Pekin, $E$.

COLOMBIE - Croix-Rouge colombienne, Carrera 7a, No. 34-65, Apartado Nacional 1110, Bogota D.E.

CONGO - Croix-Rouge du Congo, 41, av. Valcke, B.P.1712, Kinshasa.
CORÉE (République de) - Croix-Rouge de la République de Corée, 32-3 Ka Nam San Dong, Séoul.

CORÉE (République démocratique de) - CroixRouge de la République démocratique populaire de Corée, Pyongyang.

COSTA RICA - Croix-Rouge costaricienne, Calle $5^{\mathrm{a}}$ Apartado 1025, San José.

COTE D'IVOIRE - Croix-Rouge de Côte d'Ivoire, B.P. 1244, Abidjan.

CUBA - Croix-Rouge cubaine, Calle Zuluerta 471, La Havane.

DAHOMEY - Société nationale de la Croix-Rouge du Dahomey, B.P. 1, Porto-Novo.

DANEMARK - Croix-Rouge danoise, Ny Vestergade 17 , Copenhague $K$.

RÉPUBLIQUE DOMINICAINE - Croix-Rouge dominicaine, Calle Galvan, 24, Apartado 1293, Saint-Domingue.

ÉQUATEUR - Croix-Rouge équatorienne, Calle de la Cruz Roja y avenida Colombia 118, Quito.

ESPAGNE - Croix-Rouge espagnole, Eduardo Dato, 16, Madrid, 10.

ÉTATS-UNIS - Croix-Rouge américaine, National Headquarters, 17th and D. Streets, N.W., Washington 6, D.C.

ÉTHIOPIE - Croix-Rouge éthiopienne, Red Cross Road No 1, P.O. Box 195, Addis-Abéba.

FINLANDE - Croix-Rouge de Finlande, Tehtaankatu, 1 A, P.O.B. 14168, Helsinki 14.

FRANCE - Croix-Rouge française, 17, rue QuentinBauchart, Paris (8e).

GHANA - Croix-Rouge du Ghana, P.O. Box 835, Accra.

GRANDE-BRETAGNE - Croix-Rouge britannique, 14, Grosvenor Crescent, Londres, S.W.1.

GRÈCE - Croix-Rouge hellénique, Rue Lycavittou, 1, Athenes 135.

GUATÉMALA - Croix-Rouge du Guatémala, $3^{\mathrm{a}}$ Calle 8-40, Zona 1, Guatémala C.A.

HAÏTI - Croix-Rouge haĩtienne, rue Férou, B.P. 1337, Port-au-Prince.

HAUTE-VOLTA - Croix-Rouge voltalque, B.P. 340, Ouagadougou.

HONDURAS - Croix-Rouge de Honduras, Calle Henri-Dunant № 516, Tegucigalpa.

HONGRIE - Croix-Rouge hongroise, Arany Janos utca, 31, Budapest $V$.

INDE - Croix-Rouge de l'Inde, Red Cross Road 1, La Nouvelle-Delhi, 1 .

INDONÉSIE - Croix-Rouge indonésienne, Tanah Abang Barat, 66, P.O. Box 2009, Djakarta.

IRAK - Croissant-Rouge de l'Irak, Al Mansour, Bagdad.

IRAN - Société du Lion-et-Soleil-Rouge de l'Iran, Avenue Ark, Téhéran. 


\section{ADRESSES DES COMITÉS CENTRAUX}

IRLANDE - Croix-Rouge irlandaise, 16, Merrion Square Dublin 2.

ISLANDE - Croix-Rouge islandaise, Øldugotu 4, Post Box 872, Reykjavik.

ITALIE - Croix-Rouge italienne, 12, via Toscana, Rome.

JAMAÏQUE - Croix-Rouge de la Jamaïque, 76, Arnold Road, Kingsion 5.

JAPON - Croix-Rouge du Japon, 5, Shiba Park, Minato-Ku, Tokio.

JORDANIE - Croissant-Rouge jordanien, P.O. Box 1337, Amman.

KENYA - Croix-Rouge du Kénya, St. John's Gate, B.O. Box 712, Nairobi.

KOWEÏT - Croissant-Rouge de Koweït, P.O. Box 1359, Koweït.

LAOS - Croix-Rouge lao: B.P. 650, Vientiane.

LIBAN - Croix-Rouge libanaise, rue GénéralSpears, Beyrouth.

LIBÉRIA - Croix-Rouge du Libéria, National Headquaters, Corner of Tubman boulevard and $9 \mathrm{~h}$ Street Sinkor, P.O. Box 226, Monrovia.

LIBYE - Croissant-Rouge libyen, Berka Omar Mukhtar Street, P.O. Box 541, Benghazi.

LIECHTENSTEIN - Croix-Rouge du Liechtenstein, Vaduz

LUXEMBOURG - Croix-Rouge luxembourgeoise, Parc de la Ville, C.P. 234, Luxembourg.

MADAGASCAR - Croix-Rouge de la République malgache, rue Clemenceau, B.P. 1168, Tananarive.

MALAISIE - Croix-Rouge de Malaisie, Jalan Belfield 519, Kuala Lumpur.

MALI - Croix-Rouge malienne, Route de Koulikora, B.P. 280, Bamako.

MAROC - Croissant-Rouge marocain, rue Calmette, B.P. 189, Rabat.

MEXIQUE - Croix-Rouge mexicaine, Avenida Ejercito Nacional No 1032, Mexico, $10, D F$.

MONACO - Croix-Rouge monégasque, bd de Suisse, 27, Monte-Carlo.

MONGOLIE (République populaire de) - CroixRouge de la République populaire de Mongolie, Central Post Office, Post Box 537, Oulan-Bator.

NÉPAL - Croix-Rouge du Népal, Tripureswore, P.B. 217, Kathmandu.

NICARAGUA - Croix-Rouge du Nicaragua, 12, Avenida Noroeste, Managua, D.N.

NIGER - Croix-Rouge du Niger, B.P. 386, Niamey.

NIGERIA - Croix-Rouge du Nigeria, Eko Akete Close, Ikoyi, Yaba, P.O. Box 764, Lagos.

NORVÈGE - Croix-Rouge de Norvège, Parkveien, $33 \mathrm{~b}$, Oslo 3

NOUVELLE-ZÉLANDE - Croix-Rouge néo-zélandaise, 61, Dixon Street, P.O.B. 6073, Wellington, C.2.

OUGANDA - Croix-Rouge de l'Ouganda, 17, Jinja Road P.O. Box 494, Kampala.

PAKISTAN - Croix-Rouge du Pakistan, Frere Street, Karachi 4.

PANAMA - Croix-Rouge de Panama, Apartado 668, Panama.

PARAGUAY - Croix-Rouge paraguayenne, calle André Barbero y Artigas 33, Asuncion.
PAYS-BAS - Croix-Rouge néerlandaise, 27, Prinsessegracht, La Haye.

PÉROU - Croix-Rouge péruvienne, Jiron Chancay 881 , Lima.

PHILIPPINES - Croix-Rouge philippine, 860, United Nations Avenue, P.O. Box 280, Manille.

POLOGNE - Croix-Rouge polonaise, Mokotowska 14, Varsovie.

PORTUGAL - Croix-Rouge portugaise, Secrétariat général, Jardim 9 de Abril, 1-5, Lisbonne 3.

ROUMANIE - Croix-Rouge de la République Socialiste de Roumanie, Strada Biserica Amzei, 29, Bucarest.

SAINT-MARIN - Croix-Rouge de Saint-Marin, Palais gouvernemental, Saint-Marin.

SALVADOR, EL - Croix-Rouge du Salvador, $3^{a}$ avenida Norte y $3^{a}$, calle Poniente, 21, San Salvador.

SÉNÉGAL - Croix-Rouge sénégalaise, Bld. Franklin-Roosevelt, P.O.B. 299, Dakar.

SIERRA LEONE - Croix-Rouge de Sierra Leone, P.O. Box 427, 6, Liverpool Street, Freetown.

SOUDAN - Croissant-Rouge soudanais, P.O. Box 235, Khartoum.

SUĖDE - Croix-Rouge suédoise, Artillerigatan, 6, Stockholm 14.

SUISSE - Croix-Rouge suisse, Taubenstrasse, 8, Case postale 2699, 3001 Berne.

SYRIE - Croissant-Rouge syrien, 13, rue Abi-AlaAlmaari, Damas.

TANZANIE - Croix-Rouge de Tanzanie, Upanga Road, P.O.B. 1133, Dar-es-Salaam.

TCHÉCOSLOVAQUIE - Croix-Rouge tchécoslovaque, Thunovska, 18, Prague $I$.

THAÎLANDE - Croix-Rouge thallandaise, King Chulalongkorn Memorial Hospital, Bangkok.

TOGO - Croix-Rouge togolaise, 19, avenue des Alliés, B.P. 655, Lomé.

TRINITÉ-et-TOBAGO - Croix-Rouge de Trinité et-Tobago, 48, Pembroke Street, P.O. Box 357, Port of Spain.

TUNISIE - Croissant-Rouge tunisien, 19, rue d'Angleterre, Tunis.

TURQUIE - Société du Croissant-Rouge turc, Yenisehir, Ankara.

U.R.S.S. Alliance des Sociétés de la Croix-Rouge et du Croissant-Rouge de l'U.R.S.S., Tcheremushkinskij proezd 5, Moscou, W 36.

URUGUAY - Croix-Rouge uruguayenne, Avenida 8 de Octubre 2990, Montevideo.

VENEZUELA - Croix-Rouge vénézuélienne, Avenida de Andrés Bello, No. 4, Apart. 3185 , Cararas.

VIETNAM (République démocratique du) - CroixRouge de la République démocratique du Vietnam, 68, rue Bà-Trièu, Hanoi.

VIETNAM (République du) - Croix-Rouge de la République du Vietnam, rue Hông Thâp $T u$, No 201, Saigon.

YOUGOSLAVIE - Croix-Rouge yougoslave, Simina ulica broj, 19, Belgrade.

ZAMBIE - Croix-Rouge de Zambie, P.O. Box R. W. 1, Ridgeway, Lusaka. 